\title{
IBTK Haploinsufficiency Affects the Tumor Microenvironment of Myc-Driven Lymphoma in E-myc Mice
}

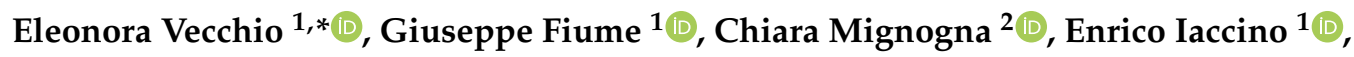 \\ Selena Mimmi ${ }^{1}$, Domenico Maisano ${ }^{1}$, Francesco Trapasso ${ }^{1}$ and Ileana Quinto ${ }^{1, *}$ \\ 1 Department of Experimental and Clinical Medicine, University Magna Graecia of Catanzaro, \\ 88100 Catanzaro, Italy; fiume@unicz.it (G.F.); iaccino@unicz.it (E.I.); mimmi@unicz.it (S.M.); \\ maisano@unicz.it (D.M.); trapasso@unicz.it (F.T.) \\ 2 Interdepartmental Service Center, University “Magna Graecia” of Catanzaro Medical School, \\ 88100 Catanzaro, Italy; mignogna@unicz.it \\ * Correspondence: eleonoravecchio@unicz.it (E.V.); quinto@unicz.it (I.Q.)
}

Received: 14 November 2019; Accepted: 28 January 2020; Published: 30 January 2020

\begin{abstract}
The tumor microenvironment is a dynamic and interactive supporting network of various components, including blood vessels, cytokines, chemokines, and immune cells, which sustain the tumor cell's survival and growth. Murine models of lymphoma are useful to study tumor biology, the microenvironment, and mechanisms of response to therapy. Lymphomas are heterogeneous hematologic malignancies, and the complex microenvironment from which they arise and their multifaceted genetic basis represents a challenge for the generation and use of an appropriate murine model. So, it is important to choose the correct methodology. Recently, we supported the first evidence on the pro-oncogenic action of IBTK in Myc-driven B cell lymphomagenesis in mice, inhibiting apoptosis in the pre-cancerous stage. We used the transgenic E $\mu$-myc mouse model of non-Hodgkin's lymphoma and Ibtk hemizygous mice to evaluate the tumor development of Myc-driven lymphoma. Here, we report that the allelic loss of Ibtk alters the immunophenotype of Myc-driven B cell lymphomas, increasing the rate of pre-B cells and affecting the tumor microenvironment in E $\mu$-myc mice. In particular, we observed enhanced tumor angiogenesis, increasing pro-angiogenic and lymphangiogenic factors, such as VEGF, MMP-9, CCL2, and VEGFD, and a significant recruitment of tumor-associated macrophages in lymphomas of $\mathrm{Ibtk}^{+/-} E \mu$-myc compared to Ibtk ${ }^{+/+} E \mu$-myc mice. In summary, these results indicate that IBTK haploinsufficiency promotes Myc tumor development by modifying the tumor microenvironment.
\end{abstract}

Keywords: tumor microenvironment; lymphoma; Myc; haploinsufficiency; heterozygous mutation; IBTK

\section{Introduction}

The chemokine system plays a key role in cancer-related inflammation involved in all stages of tumor development. Noteworthy, chronic inflammation in the tumor microenvironment contributes to the progression of many cancer types [1-3]. It is widely recognized that chronically activated immune cells sustain tumor growth and survival. However, the underlying mechanisms of this connection remain unclear. The tumor microenvironment consists of different cell populations, including fibroblasts, endothelial cells, and immune cells [4,5]. It is now clear that chronically activated immune cells can promote tumor growth and facilitate tumor survival. Macrophages are typically the main inflammatory component, but a variety of tumor-infiltrated immune cells can participate in tumor promotion [6]. In general, the presence of macrophages in tumor tissues confers a poor 
prognosis [7]. Furthermore, angiogenesis is also a critical process in malignant progression [8]. Tumor growth is strictly associated with the expansion of vasculature that supplies oxygen and nutrients to the tumor tissue and allows metastatic spread to colonize distant organs. It was shown that the tumor microenvironment (TME) composition as well as its functionality are tissue dependent and context dependent [9]. A useful approach to understand the impact of TME on tumor evolution is the genetically engineered mouse model (GEMM). GEMMs are a powerful tool to reveal multiple genes implicated in the genesis of lymphoma and are extensively used to validate oncogene or tumor suppressor function $[10,11]$. Whereas each mouse model may clarify the molecular mechanism of lymphoma, evaluation schema for mouse tumors have not yet been standardized [10].

Myc is a transcription factor regulating the expression of genes involved in cell growth and metabolism, and it is one of the most frequently dysregulated transcription factors in human malignancies [12]. Over expression of Myc is a well-characterized initiating step in human Burkitt's lymphoma, a non-Hodgkin's lymphoma [13]. The E $\mu$-myc transgenic mouse is a pre-clinical model of human non-Hodgkin's lymphoma, which develops aggressive B cell-derived lymphomas at an early age, with a $90 \%$ mortality rate by 20 weeks of age and median age of death at 12 weeks $[13,14]$. $\mathrm{Myc}^{\mathrm{Tg}}$ lymphomas develop from the B220 ${ }^{\mathrm{low}}$ pre-B and B cell populations, and Ig gene rearrangement analyses indicate that most are monoclonal $[13,15]$. Lymphomas developed from $E \mu$-myc transgenic mice present increased blood and lymphatic vascular growth in secondary lymphoid organs [16].

The human IBTK gene is involved in the stress response and tumor growth $[17,18]$. It expresses a main protein IBtk $\alpha$ isoform, encoding a substrate receptor of Cullin3 ligase that promotes the proteasome-associated degradation of tumor repressor PDCD4 [19]. IBTK RNA interference affected the wide genome expression and RNA splicing in a cell-type-specific manner [20]. IBTK was hyper-expressed in chronic lymphocytic leukemia correlating with disease progression, and it was required for B cell survival upon stress induced by chemotherapeutic agents [21]. Based on the strong homology between the human and murine IBTK gene, we previously developed Ibtk knockout mice to address the role of IBTK in B-lymphomagenesis [22]. By taking advantage of the E $\mu$-myc transgenic mouse, we generated $I b t k^{-/} E \mu$-myc offspring to support the first evidence of the pro-survival action of IBTK in Myc-dependent B-lymphomagenesis counteracting apoptosis of pre-cancerous B-cells [22]. In the present study, the IBTK haploinsufficiency alters tumor development and, consequently, the tumor microenvironment by enhancing tumor vascularization in Myc-driven B cell lymphoma. Allelic loss of IBTK promotes the expression of pro-angiogenic and inflammatory cytokines as VEGF family proteins together with the recruitment of tumor-associated macrophages (TAMs) as immune cells in Myc-driven lymphoma. These results contribute to the characterization of IBTK as a novel regulator gene of the tumor microenvironment.

\section{Results}

\subsection{IBTK Haploinsufficiency Increases the Size and Vascularization of Spleen and Lymph Nodes of E $\mu$-myc Tumor Mice}

$E \mu$-myc transgenic mice are widely used as a preclinical model of Myc-dependent B-lymphomagenesis $[13,14]$. We previously investigated the contribution of IBTK to malignant transformation of B cells by crossing E $\mu$-myc mice with $\mathrm{Ibtk}^{--}$mice to generate $\mathrm{Ibtk}^{-/} E \mu$-myc offspring [22]. While the complete loss of $I b t k$ ( $I b t k^{--} E \mu$-myc mice) delayed the lymphoma onset and increased the lifespan, the loss of a single allele of $I b t k\left(I b t k^{+-} E \mu\right.$-myc mice) did not significantly affect tumor onset and the median age of mortality in $E \mu$-myc mice littermates [22].

In the present study, we addressed the question of whether the loss of a single Ibtk allele could still have some effects on lymphoma growth. The reduced IBTK gene expression was confirmed in $I b t k^{+/-} E \mu-m y c$ compared to Ibtk $k^{+/+} E \mu-m y c$ mice, as measured by real-time PCR (Figure 1A,B). At the macroscopic level, a significant increase in the weight and volume of lymph nodes (Figure 1C,D,E) and spleen (Figure 1F,G,H) was observed in a cohort of 12- to 16-week-old $I b t k^{+/-} E \mu$-myc mice compared to 
$I b t k^{+/+} E \mu-m y c$ mice, after tumor onset. We also observed the increased vascularization and hemorrhages of tumor lymph nodes of $I b t k^{+/} E \mu-m y c$ compared to $I b t k^{+/+} E \mu-m y c$ mice (Figure 1E).
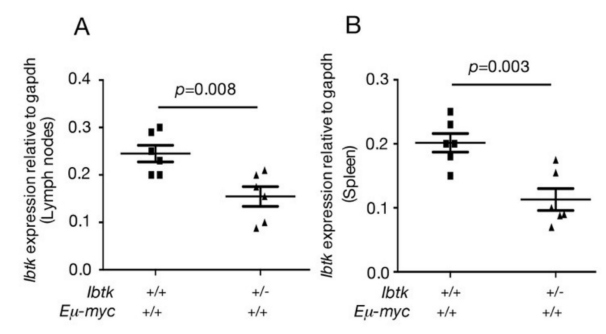

C

D
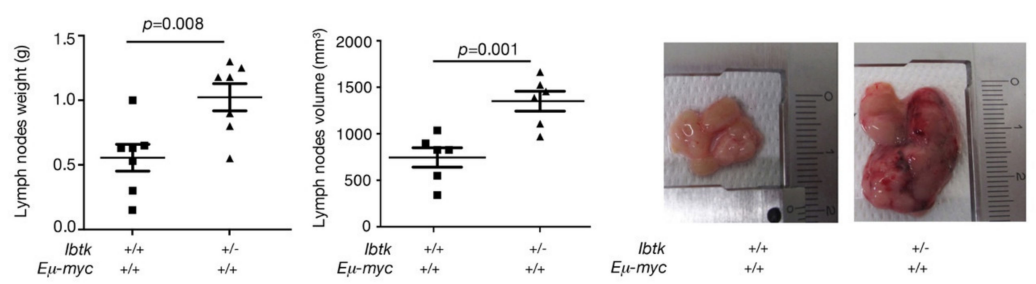

$\mathrm{F}$

$\mathrm{G}$

$\mathrm{H}$
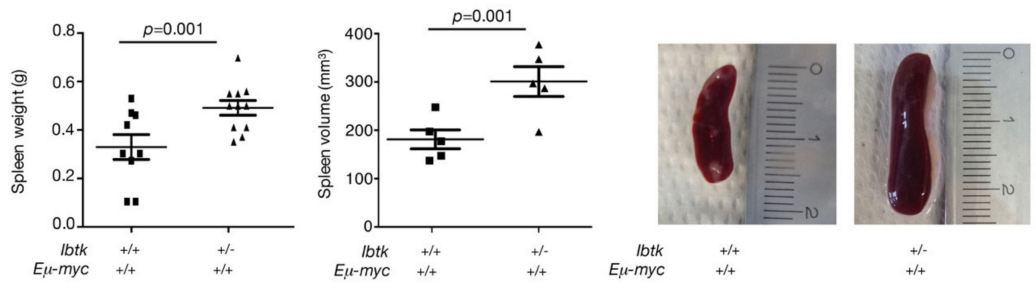

Figure 1. IBTK haploinsufficiency promotes the enlargement of tumor lymph nodes and spleen in E $\mu$-myc mice. A,B. Ibtk mRNA levels were measured by RT-PCR in the tumor lymph nodes and spleen of $\mathrm{Ibtk}^{+/+} E \mu-m y c$ and $\mathrm{Ibtk^{+/ }} E \mu-m y c$ mice, and normalized to Gapdh mRNA. C. Weights of lymph nodes of $I b t k^{+/+} E \mu-m y c$ and $I b t k^{+/-} E \mu-m y c$ sick mice. Values are the mean \pm SEM ( $n=7 /$ genotype). D. Volume of lymph nodes of $I b t k^{+/+} E \mu-m y c$ and $I b t k^{+/-} E \mu-m y c$ sick mice. Values are the mean \pm SEM ( $n=6 /$ genotype). E. Representative morphology of tumor lymph nodes. Scale bar is indicated. F. Weights of spleens of sick lymphoma-burdened $I b t k^{+/+} E \mu-m y c$ and Ibtk ${ }^{+/-} E \mu-m y c$ mice. Values are the mean \pm SEM ( $n=10$ /genotype). G. Volume of spleens of sick lymphoma-burdened Ibtk $k^{+/+} E \mu-m y c$ and $I b t k^{+-} E \mu$-myc mice. Values are the mean \pm SEM ( $n=5$ /genotype) H. Representative morphology of tumor spleens. Scale bar is indicated.

By flow cytometry, we performed tumor immunophenotyping of lymphoma cells using B220, IgM, and $\mathrm{IgD}$ as markers of B-cell subpopulations. $\mathrm{Ibtk}^{+/+} E \mu$-myc mice developed $60 \%$ pre-B lymphoma $\left(\mathrm{B}_{22}{ }^{+} \operatorname{IgM}{ }^{-} \operatorname{IgD}^{-}\right), 35 \%$ mature B lymphoma $\left(\mathrm{B}^{2} 20^{+} \operatorname{IgM}^{+} \operatorname{IgD}^{+}\right)$, and $5 \%$ pre-B/B lymphomas (Table 1$)$, which was consistent with the tumor immunophenotype reported by previous studies $[13,23,24]$. Otherwise, $I b t k^{+-} E \mu-m y c$ mice showed a significant increase of pre-B lymphoma (96\%) and a decrease of mature B lymphoma (1\%) (Table 1). These results indicate that the loss of a single Ibtk allele raises the size of Myc-driven lymphoma, with a significant impact on the tumor immunophenotype, with pre-B cell lymphoma being a more aggressive lymphoma.

Consistent with the increased vascularization (Figure 1E), the vascular density of tumor lymph nodes was higher in $I b t k^{+/} E \mu$-mycthan $I b t k^{+/+} E \mu$-mycmice, as shown by immunofluorescence staining of the blood vessel specific marker CD31 (Figure 2A,B). The number of lymphatic vessels was also increased in the tumor lymph nodes of $I b t k^{+/-} E \mu$-myccompared to $\mathrm{Ibtk}{ }^{+/+} E \mu$-mycmice, as stained with the lymphatic vessel marker LYVE-1 (Figure 2C,D). A massive presence of necrotic areas was observed in the tumor lymph nodes of $I b t k^{+/} E \mu-m y c c o m p a r e d ~ t o ~ I b t k^{+/+} E \mu-m y c m i c e$ (Figure 2E,F). By hematoxylin/eosin 
staining, we also confirmed increased vascularization in the spleen of $I b t k^{+/} E \mu-m y c c o m p a r e d$ to $I b t k^{+/+} E \mu$-mycmice (Figure 2G,H). These results indicate that IBTK haploinsufficiency increases the vascularization of the tumor lymph nodes and spleen in $E \mu$-mycmice, which is associated with severe tumor necrosis. Previous studies on young preneoplastic and tumor E $\mu$-myc mice showed increased growth of functional blood and lymphatic vessels in hematolymphoid tissues relative to normal littermate control mice, with this effect being partly dependent on VEGF production by B lymphocytes [16]. Thus, we analyzed the VEGF expression level in lymphoma tissues by immunofluorescence. VEGF was highly expressed in the tumor lymph nodes of $I b t k^{+-} E \mu$-myccompared to $I b t k^{+/+} E \mu$-mycmice (Figure 2I). By immunoblotting, we observed a significant VEGF increase in the protein extracts of B-cells isolated from the tumor lymph nodes of $I b t k^{+/} E \mu$-myccompared to $I b t k^{+/+} E \mu-m y c m i c e$ (Figure 2J). Altogether, these results could indicate that the loss of a single $I b t k$ allele enhances the production of VEGF in B lymphoma cells, as a mechanism to induce vessel growth.

Table 1. Immunophenotype of lymphomas developed by $I b t k^{+/+} E \mu-m y c$ and $I b t k^{+/} E \mu-m y c$ mice.

\begin{tabular}{cccc}
\hline \multirow{2}{*}{ Genotype } & Pre-B cell & Pre-B/B cell & B cell \\
\cline { 2 - 4 } & lymphoma & lymphoma & lymphoma \\
\hline$I b t k^{+/+} E \mu-m y c(n=20)$ & $12(60 \%)$ & $1(5 \%)$ & $7(35 \%)$ \\
$I b t k^{+/} E \mu-m y c(n=24)$ & $23(96 \%)$ & $0(0 \%)$ & $1(4 \%)$ \\
\hline
\end{tabular}

Cell suspensions from lymphomas were stained with the antibodies against B220, IgM, and IgD, and analyzed by flow cytometry.

2.2. IBTK Haploinsufficiency Increases the Production of Pro-Angiogenic Factors and Cytokines in the Tumor Microenvironment of E $\mu$-myc Mice

Tumor-associated angiogenesis is a complex process, involving many proangiogenic factors, such as cytokines and chemokines. Thus, we asked the question of whether the haploidy of Ibtk could affect the variation of the expression of angiogenic factors on tumor cells. To this end, we analyzed the mRNA expression of 84 genes involved in the angiogenesis pathway by quantitative real-time PCR of total RNA extracted from the tumor lymph nodes of both genotypes (Table S1). The expression of 51 genes was significantly upregulated in $I b t k^{+/-} E \mu$-mycmice, including VEGFD, VEGFR1 (as a receptor of VEGF family proteins), MMP9, and CCL2 (Figure 3A,B). Noteworthy, the highest expressed cytokine observed was VEGFD. This is a vascular endothelial growth factor previously identified as one of the predominantly lymphangiogenic factors, inducing lymphangiogenesis in transgenic mouse models [25]. This result confirms increased tumor lymphatic vascularization of $I b t k^{+-} E \mu-m y c c o m p a r e d ~ t o ~ I b t k^{+/+}$ $E \mu$-mycmice as shown by immunofluorescence with the specific lymphangiogenic marker Lyve1 [26] (Figure 2C). The enhanced expression of pro-angiogenic factors was consistent with the increased vascularization of tumor lymph nodes of $I b t k^{+/-} E \mu$-myccompared to $I b t k^{+/+} E \mu$-mycmice. Next, we extended our study to the tumor microenvironment. The latter includes various cell types, extracellular matrix, growth factors, proteolytic enzymes, and signaling molecules, all of which contribute to cancer development and progression. In particular, the chronic inflammation driven by chemokines and cytokines at the tumor site promotes tumor progression [27]. Thus, we sought to evaluate whether the loss of a single Ibtk allele could affect chemokine and cytokine expression in the tumor microenvironment of Myc-dependent lymphomas. By cytokine array, we tested the expression levels of 31 cytokines in the protein lysates of lymphomas (Table S2). We confirmed that Pro-MMP9, VEGF, and VEGFD were the cytokines more significantly increased in $I b t k^{+/-} E \mu$-myccompared to Ibtk ${ }^{+/+} E \mu$-mycmice. (Figure 4 A-C) (Table S2). Among the cytokines related to the tumor microenvironment, these are mainly involved in angiogenesis, promoting tumor growth [28,29]. Previous studies showed that CCL2 regulates the trafficking of monocytes, macrophages, and other inflammatory cells [30]. CCL2 also promotes the recruitment of tumor-associated macrophages (TAMs) that may secrete VEGF and the proteolytic enzyme MMP-9, which is involved in the vascularization of tumor tissues [31]. TAMs act through the promotion of angiogenesis and the facilitation of tumor metastasis [32]. Consequently, we confirmed the presence of CCL2 in lymphoma tissues by immunoblotting. We observed both increased CCL2 
mRNA (Figure 3A,B) and protein (Figure $4 \mathrm{D}$ ) expression from tumor lymph nodes of $\mathrm{Ibtk}^{+-} \mathrm{E \mu}-\mathrm{myc}$

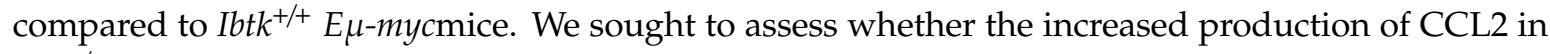
$I b t k^{+-} E \mu$-myclymphomas could affect the presence of TAMs. By flow cytometry, a significant increase of TAMs in lymphoma of $I b t k^{+-} E \mu$-myccompared to $I b t k^{+/+} E \mu$-mycmice was observed (Figure 4E).
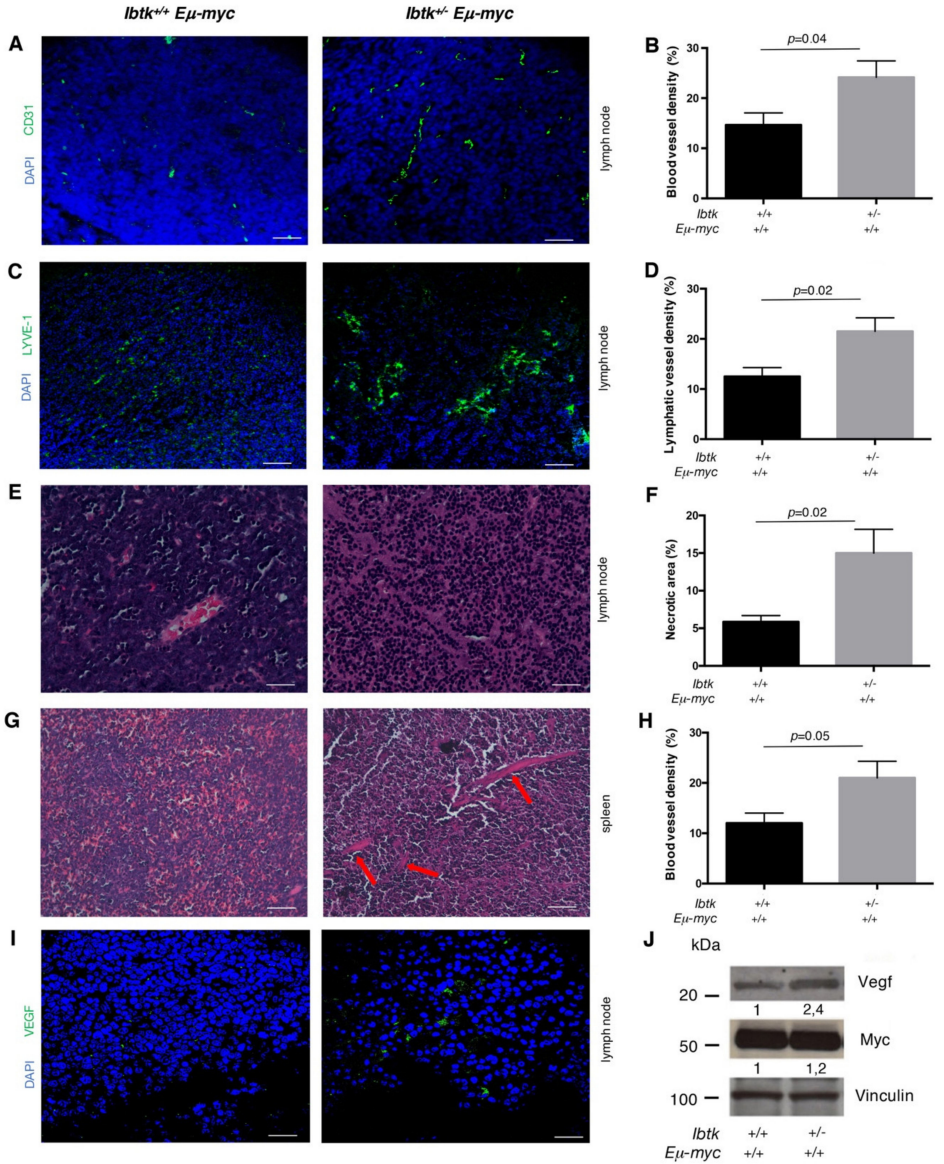

Figure 2. IBTK haploinsufficiency results in increased tumor vascularization and tissue necrosis. A. Sections of tumor lymph nodes of $I b t k^{+/+} E \mu-m y c$ and $I b t k^{+/-} E \mu-m y c$ mice stained for CD31 (green) to visualize blood vessels, and analyzed by confocal microscopy. A representative image is shown. Scale bars: $150 \mu \mathrm{m}$. Magnification 200×. B. Quantitation of blood vessel density in cancerous lymph nodes of $I b t k^{+/+} E \mu-m y c(n=6)$ and $I b t k^{+-} E \mu-m y c(n=6)$ mice. Values are the mean \pm SEM. C. Sections of tumor lymph nodes of $\mathrm{Ibtk^{+/+ }} E \mu-m y c$ and $\mathrm{Ibtk}^{+/} E \mu-m y c$ mice stained for LYVE-1 (green) to visualize lymphatic vessels, and analyzed by confocal microscopy. A representative image is shown. Scale bars: $150 \mu \mathrm{m}$. Magnification 200x. D. Quantitation of the lymphatic vessel density in the tumor lymph nodes of $I b t k^{+/+} E \mu-m y c(n=6)$ and $I b t k^{+/-} E \mu-m y c(n=6)$ mice. Values are the mean \pm SEM. E. Sections of tumor lymph nodes stained by hematoxylin/eosin. Representative images of tumor tissues are shown. Scale Bars: $75 \mu \mathrm{m}$. Magnification 400×. F. Quantitation of necrosis levels was estimated as a percentage of the total area of tumor lymph nodes of $I b t k^{+/+} E \mu-m y c(n=6)$ and Ibtk $k^{+-} E \mu-m y c(n=6)$ mice. Values are the mean \pm SEM. G. Spleen sections of sick $I b t k^{+/+} E \mu-m y c$ and $I b t k^{+/-} E \mu-m y c$ mice stained by hematoxylin/eosin. Red arrows indicate marked vascularization in $I b t k^{+/-} E \mu-m y c$ mice. Scale bars: 150 $\mu \mathrm{m}$. Magnification 200×. H. Quantitation of the vessel density in the spleen of $I b t k^{+/+} E \mu-m y c(n=6)$ and $I b t k^{+/} E \mu-m y c(n=6)$ sick mice. Values are the mean \pm SEM. I. Sections of tumor lymph nodes of $I b t k^{+/+} E \mu-m y c$ and $I b t k^{+-} E \mu-m y c$ mice stained for VEGF (green) and analyzed by confocal microscopy. A representative image is shown. Scale Bars: $50 \mu \mathrm{m}$. Magnification $630 \times$. J. Immunoblot analysis of VEGF, Myc, and vinculin expression in the protein extracts of B cells isolated from the tumor lymph nodes of $I b t k^{+/+} E \mu-m y c$ and $I b t k^{+/} E \mu-m y c$ mice. Protein bands were normalized to the corresponding vinculin intensity. 
A

\begin{tabular}{|c|c|c|}
\hline Gene & Fold change & p-value \\
\hline VEGF D & 5.43 & 0.0006 \\
\hline TGFA & 4.83 & 0.000015 \\
\hline NOS3 & 4.82 & 0.00015 \\
\hline VEGFRI & 4.8 & 0.00097 \\
\hline PLG & 4.78 & 0.001 \\
\hline TIE1 & 4.68 & 0.00015 \\
\hline IL6 & 4.52 & 0.00016 \\
\hline Timp1 & 4.46 & 0.0011 \\
\hline F3 & 4.42 & 0.0003 \\
\hline ANGPT1 & 4.39 & 0.0001 \\
\hline TBXI & 4.38 & 0.0002 \\
\hline $\mathrm{CSF} 3$ & 4.33 & 0.0003 \\
\hline PGF & 4.33 & 0.00095 \\
\hline TYMP-1 & 4.28 & 0.0007 \\
\hline BAII & 4.09 & 0.0002 \\
\hline TEK & 4.1 & 0.0002 \\
\hline EDN1 & 3.99 & 0.00004 \\
\hline CXCL5 & 3.92 & 0.0003 \\
\hline SERPINE1 & 3.92 & 0.00001 \\
\hline ERBB2 & 3.94 & 0.00002 \\
\hline COL18AI & 3.86 & 0.0004 \\
\hline $\mathrm{F} 2$ & 3.83 & 0.0002 \\
\hline THBS2 & 3.75 & 0.0008 \\
\hline LEP & 3.69 & 0.0003 \\
\hline FGFR3 & 3.69 & 0.0005 \\
\hline EPHB4 & 3.63 & 0.000035 \\
\hline FGF6 & 3.58 & 0.0001 \\
\hline TGFB3 & 3.57 & 0.0007 \\
\hline PTGSI & 3.55 & 0.004 \\
\hline FGF2 & 3.43 & 0.0007 \\
\hline LECT1 & 3.31 & 0.0002 \\
\hline HGF & 3.21 & 0.00001 \\
\hline SPHK1 & 3.25 & 0.00015 \\
\hline CCL11 & 3.14 & 0.001 \\
\hline JAGI & 2.96 & 0.0002 \\
\hline COL4A3 & 2.90 & 0.003 \\
\hline EFNB2 & 2.88 & 0.002 \\
\hline KDR & 2.86 & 0.0006 \\
\hline EPASI & 2.84 & 0.0008 \\
\hline FGF1 & 2.82 & 0.003 \\
\hline CCL2 & 2.84 & 0.0003 \\
\hline CXCL1 & 2.67 & 0.0002 \\
\hline ITGB3 & 2.63 & 0.0003 \\
\hline MMP9 & 2.62 & 0.0005 \\
\hline $\mathrm{CDH} 5$ & 2.58 & 0.0007 \\
\hline MMP2 & 2.33 & 0.0003 \\
\hline THBSI & 2.19 & 0.0001 \\
\hline TNFSF12 & 2.12 & 0.008 \\
\hline VEGF B & 2.01 & 0.005 \\
\hline IGFI & 2.01 & 0.002 \\
\hline
\end{tabular}

B

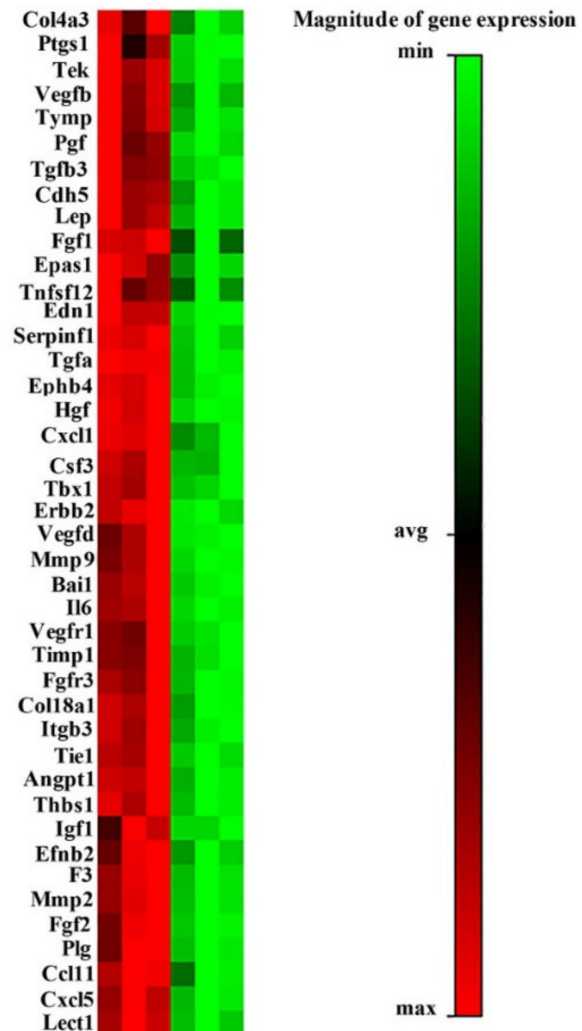

Figure 3. Increased expression of pro-angiogenic genes in the tumor lymph nodes of Ibtk ${ }^{+-} E \mu$-myc mice. A. Total RNA was extracted from the tumor lymph nodes of $I b t k^{+/+} E \mu-m y c$ and $I b t k^{+/} E \mu-m y c$ mice, and analyzed by quantitative real-time PCR using SABiosciences Angiogenesis RT ${ }^{2}$ Profiler RT-PCR array. A difference in gene expression was accepted at more than a 2-fold increase. Upregulated genes are indicated as the fold change of $I b t k^{+/} E \mu-m y c$ relative to $I b t k^{+/+} E \mu-m y c$ mice. Data were analyzed by two-tailed unpaired Student ${ }^{\prime} t$ test $(p$ value $<0.05)$. B. Heat-map of the angiogenesis-related gene expression profile in $\mathrm{Ibtk^{+/- }} E \mu$-myc compared to $\mathrm{Ibtk^{+/+ }} E \mu$-myc tumor B-cells. 
A

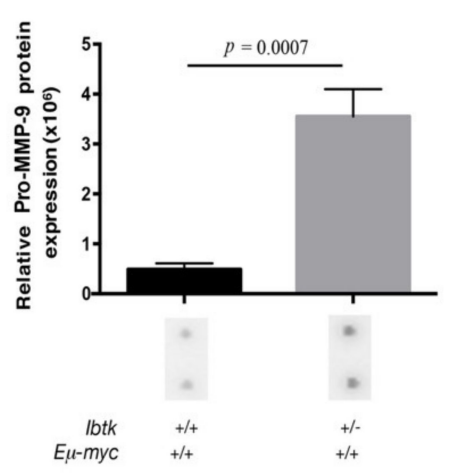

D

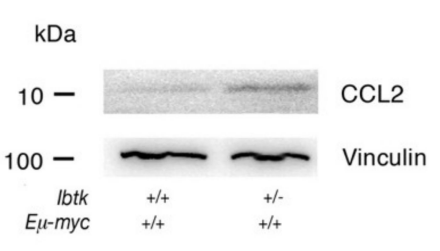

B

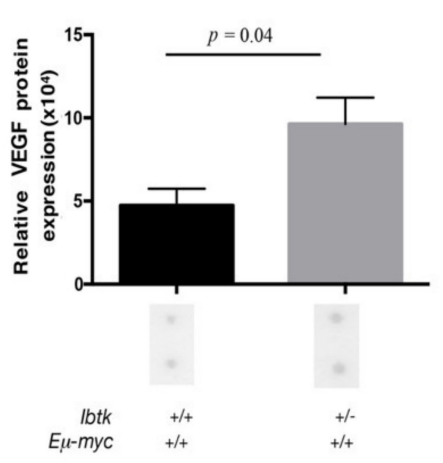

E

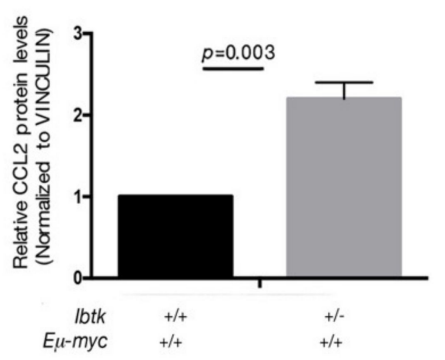

C

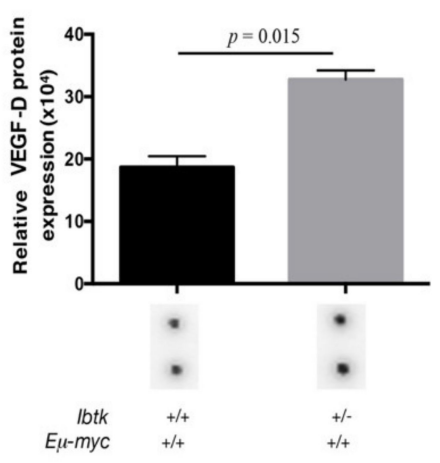

F

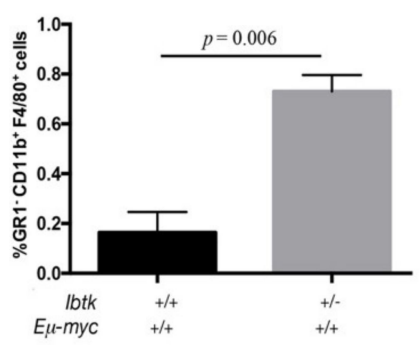

Figure 4. Cytokine expression and recruitment of tumor-associated macrophages in the tumor lymph nodes of $I b t k^{+/+} E \mu-m y c$ and $I b t k^{+/} E \mu-m y c$ mice. A, B, C. Bar diagram showing the quantification of pro-MMP9, VEGF, and VEGF-D protein expression levels from Ibtk ${ }^{+/+} E \mu-m y c$ and $I b t k^{+/-} E \mu-m y c$ cancerous mice. Densitometry data are extracted, the background was subtracted, and the data were normalized to the positive control signals, according to the manufacturer's protocols. Values are the mean \pm SEM ( $n=3$ /genotype). Representative images of individual cytokine spots are shown from identical exposures. D. Immunoblot analysis of CCL2 and vinculin expression in the protein extracts of tumor cells from $I b t k^{+/+} E \mu-m y c$ and $I b t k^{+/} E \mu-m y c$ mice. Protein bands were normalized to the corresponding vinculin intensity. E. Bar diagram showing quantitation of CCL2 protein. Protein bands were measured by densitometry as arbitrary units and normalized to vinculin as the internal control. Values are the mean \pm SEM ( $n=3 /$ genotype). F. Cell suspensions of tumor lymph nodes were stained with fluorescent-conjugated antibodies to reveal tumor-associated macrophages (GR1- CD11b+ F4/80+ cells) and analyzed by flow cytometry. Values are the mean $\pm \operatorname{SEM}(n=3 /$ genotype).

Taken together, these results suggest the potential involvement of IBTK in both enhanced tumor angiogenesis and TAM infiltration within the tumor.

\section{Discussion}

In this study, we analyzed the effect of IBTK haploinsufficiency in B-lymphoma by taking advantage of E $\mu$-myc transgenic mice, a preclinical model of non-Hodgkin's lymphoma. We showed that the loss of a single Ibtk allele increases the tumor mass and affects the tumor microenvironment of $E \mu$-myc mice. IBTK haploinsufficiency induces enlargement of the spleen and lymph nodes, together with an increased rate of pre-B cell lymphoma. Additionally, we observed a significant increase in pro-angiogenic and lymphangiogenic cytokines associated with TAM infiltration of lymphomas in $I b t k^{+/-}$E $\mu$-myc compared to $I b t k^{+/+} E \mu-m y c$ mice.

In previous reports, both young pre-neoplastic and older tumor $E \mu$-myc mice showed increased growth of functional blood and lymphatic vessels of hemato-lymphoid tissues relative to their normal littermate control mice. Moreover, the enhanced vascularization was associated with VEGF production by B-lymphocytes, suggesting a tumor-dependent mechanism of vascularization mediated by VEGF [16]. 
Here, we report that the allelic deletion of $I b t k$ raises the vascularization of tumor lymph nodes and spleen, as shown by immunohistological staining. This effect was associated with an increased production of VEGF as measured by the immunofluorescence of lymphoma tissues and Western blotting of tumor B cells. Altogether, these data suggest that IBTK haploinsufficiency contributes to upregulating VEGF expression and, consequently, promoting vessel growth in Myc-dependent lymphoma. We also observed an increased necrosis of tumor lymph nodes of $I b t k^{+/-} E \mu-m y c$ compared to $I b t k^{+/+} E \mu-m y c$ mice. These findings are in agreement with previous reports, correlating necrosis of breast carcinoma with total vessel counts in highly vascularized tumor areas [33].

In addition to VEGF, the lymphoma tissues of $I b t k^{+-} E \mu-m y c$ mice hyperexpressed other pro-angiogenic factors that could induce vascularization. In particular, the expression of chemokine CCL2 was significantly elevated in the B-lymphoma cells of $I b t k^{+-} E \mu-m y c$ mice. CCL2 is a main monocyte-recruiting chemokine that acts through binding to the CCR2 receptor expressed on immune cells [34]. The enhanced expression of CCL2 was reported in many types of cancer, including multiple myeloma, melanoma, esophageal, gastric, colorectal, lung, breast, ovary, and prostate cancer [30,34]. Of note, CCL2 contributes to angiogenesis by attracting M2-like macrophages, so-called TAMs, which secrete pro-angiogenic cytokines, such as VEGF and the proteolytic enzymes, MMP-2 and MMP-9 [34-37]. According to these observations, we found an increased production of CCL2 that we could associate with the increased presence of TAMs in the lymphomas of Ibtk ${ }^{+-} E \mu-m y c$ compared to $I b t k^{+/+} E \mu-m y c$ mice. Thus, the increased lymphoma vascularization of $I b t k^{+/-} E \mu-m y c$ mice could be a consequence of the upregulated CCL2/TAMs/VEGF network. In this regard, the correlation between TAM infiltration and angiogenesis was previously shown in different human cancers, including breast cancer, melanoma, glioma, gastric cancer, B-cell non-Hodgkin's lymphoma, mucoepidermoid carcinoma of salivary glands, and leiomyosarcoma, and it was associated with poor clinical outcomes [38]. Based on our observations in Ibtk $k^{+-} E \mu-m y c$ mice, IBTK could represent a novel modifier gene of the tumor microenvironment, acting through the deregulation of angiogenic and inflammatory factors.

A deeper knowledge of the interaction between cancer cells and the microenvironment is critical for understanding tumor pathogenesis and potential new therapeutic targets. Different strategies have been developed to reduce angiogenesis to counteract tumor progression. For example, VEGF-dependent pathways have been considered a target of tumor therapy [39]. However, while some cancer types have shown an effective therapeutic response, the benefits of anti-angiogenic agents have been revealed to be transient and followed by tumor relapse [39-41]. With IBTK being required for cancer B cell survival [21,22] and lymphoma vascularization (this study), the combination of IBTK-targeting therapies with anti-angiogenic agents could improve the therapeutic efficacy.

\section{Materials and Methods}

\subsection{Mice}

E $\mu$-myc transgenic mice $(\mathrm{TgN}(\mathrm{IghMyc}) 22 \mathrm{Bri} / \mathrm{J})$ were obtained from The Jackson Laboratory (Bar Harbor, Maine; USA). Ibtk $k^{+/-}$mice were obtained by mating $I b t k^{+/+}$and $I b t k^{-/-}$mice [22]. Subsequently,

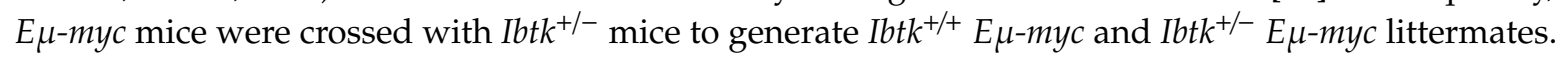
Mice were monitored daily for signs of morbidity and tumor development. Moribund mice and mice with obvious tumors were sacrificed, and single-cell suspensions were obtained from tumor tissues and frozen in $10 \%$ DMSO for RNA and protein analyses.

The $E \mu$-myc transgene was detected as a 600 -bp product by genomic PCR amplification, according to a previously described protocol [42]. Genotyping of Ibtk and $\beta g e o$ genes was performed with primers 5'-GATGTAAAGCCGTGGGAGAA-3' and 5'-ATGTGGAGAGGAGGCAGAGA-3' (800bp product), and 5'-GATGTAAAGCCGTGGGAGAA- $3^{\prime}$ and $5^{\prime}$-CACTCCAACCTCCGCAAACTC-3' (500bp product), respectively [22]. Mice were sacrificed at 6-8 weeks after tumor onset, and the volume of lymph nodes was measured by the length and width. The lymph node volume was calculated by 
the formula: Length $\times \mathrm{Width}^{2} / 2$, and expressed as $\mathrm{mm}^{3}$ [43]. The volume of the spleen was calculated by the formula: Length $\times$ Width $\times$ Height, and expressed as $\mathrm{mm}^{3}$ [44]. The Bioethical Committee of the University Magna Graecia of Catanzaro approved the experimental protocols. Animal experiments were carried out in accordance with the protocol n.794/2016-PR approved by the Italian Ministry of Health.

\subsection{Histological Analysis}

For analysis of the intra-tumoral micro vessel density, $10-\mu \mathrm{m}$ tissue sections were obtained by cryostat (Leica Biosystems Inc., Buffalo Grove, IL USA), incubated with monoclonal anti-CD31 antibody (BD Biosciences, San Jose, CA, USA), anti-VEGF (Santa-Cruz, TX, USA), anti-LYVE1 (BD Biosciences), and DAPI (SIGMA, St. Louis, MO, USA), and examined by confocal microscopy (Leica TC-SP2) [45]. Microvessel density within tumor sections was determined at a 200× magnification in five fields of each tumor section, as described [46]. Microvessel density was reported as the mean number of microvessels per field $\left(56.25 \mu \mathrm{m}^{2}\right)$. For the histopathological analysis, lymph nodes and spleen were formalin-fixed and embedded in paraffin, and 5- $\mu \mathrm{m}$ tissue sections were stained with hematoxylin and eosin. Tissue sections were examined with a Leica Microscope (Leica Microsystems GmbH, Wetzlar, Germany) equipped with $10 \times, 40 \times$, and $100 \times$ objective lenses.

\subsection{Western Blot Analysis and Cytokine Antibody Arrays}

Cells and tissues were lysed in ice-cold modified RIPA buffer (10 mM Tris-HCl, pH 7.5, $150 \mathrm{mM}$ $\mathrm{NaCl}, 1 \mathrm{mM}$ EDTA, 1\% Igepal), as previously described [47]. Protein samples were separated by electrophoresis on NuPAGE $4 \%-12 \%$ polyacrylamide gel (Thermo Fisher Scientific, Waltham, MA, USA) and transferred to a nitrocellulose membrane (BioRad, Hercules, CA, USA). Equal amounts of protein were Western blotted using the following antibodies: Myc (\#5605, Cell Signaling Technology, Massachusetts, USA), VEGF (sc-507, Santa-Cruz, Texas, USA), CLL2(\#2029, Cell Signaling Technology, Massachusetts, USA), and Vinculin (V9131, Sigma-Aldrich). For the cytokine array, whole tumor lysates were collected to test the cytokine levels as outlined in the mouse cytokine C-Series antibody array protocol (RayBiotech, Georgia, USA). Relative cytokine contents were quantified using Uvitec densitometry software. Briefly, after the raw numerical densitometry data were extracted, the background was subtracted and the data were normalized to the positive control signals, according to the manufacturer's protocols. Data were analyzed using the RayBio Analysis Software Tools.

\subsection{Real Time PCR, Data Normalization, and Analysis}

Total RNA was isolated from the tumor lymph nodes of $I b t k^{+/+} E \mu-m y c$ or $I b t k^{+-} E \mu-m y c$ mice using GenElute Mammalian Total RNA Miniprep reagent (Sigma). After DNase treatment, cDNA was synthesized by an $\mathrm{RT}^{2}$ First Strand kit (SABiosciences. MD, USA) according to the company's instructions. Gene expression profiling was performed using the Angiogenesis $\mathrm{RT}^{2}$ Profiler PCR Array (SABiosciences, MD, USA). This platform is designed to profile the expression of 84 genes of angiogenesis; for a comprehensive list of genes, see http://www.sabiosciences.com. Quantitative reverse transcriptase PCR (RT-PCR) was performed using an iScript RT-PCR System (Biorad, CA, USA) according to the manufacturer's instructions. Relative gene expression was determined using the $\Delta \Delta \mathrm{Ct}$ method. Data were further analyzed using the PCR Array Data Analysis Web Portal (http://www.SABiosciences.com/pcrarraydataanalysis.php). The genes $\beta 2$-microglobulin (B2M), Heat shock protein 90 alpha (cytosolic), class B member 1 (Hsp90ab1), glucuronidase- $\beta$ (Gusb), glyceraldehyde-3-phosphate dehydrogenase (GAPDH), and $\beta$-actin (ACTB) were included in the PCR array as endogenous controls for normalization. Each replicate cycle threshold (CT) was normalized to the average $\mathrm{CT}$ of the five endogenous controls. The following formula was used to calculate the amount of transcripts in the samples relatively to the control group, both of which were normalized to the endogenous controls: $\Delta \Delta \mathrm{Ct}=\Delta \mathrm{Ct}\left(\mathrm{Ibtk^{+/- }} E \mu\right.$-myc as test sample) $-\Delta \mathrm{Ct}$ (Ibtk ${ }^{+/+} E \mu-m y c$ as control sample). $\Delta \mathrm{Ct}$ is the log2 difference in $\mathrm{Ct}$ between the test sample gene and the control sample by 
subtracting the average $\mathrm{Ct}$ of control genes from each replicate. The fold-change of each sample relative to the control sample $=2^{-\Delta \Delta \mathrm{Ct}}$. The gene expression change between $I b t k^{+/-} E \mu-m y c$ and Ibtk $k^{+/+} E \mu-m y c$ tumor cells was reported as a fold increase or decrease. The adopted criteria included the following: a) Student's $t$ test with $p$ value $<0.05$; and b) mean difference equal to or greater than 2 -folds change in expression levels. The statistical analysis was based on the web-based program for Profiler TM PCR Array Data Analysis. Data were evaluated as the mean of at least three independent experiments.

\subsection{Isolation of $B$ Cells}

The separation of B cells was performed by depletion of non-B cells using magnetic-activated cell sorting (MACS) B-cell isolation kit or by CD19 MicroBeads and MS columns (Miltenyi Biotech, Bergisch Gladbach, Germany) according to the manufacturer's protocols. As a control, flow cytometry of MACS-separated cells revealed a $95 \%$ purity of B cells.

\subsection{Flow Cytometry}

Lymphoma immunophenotype was assessed as previously described [22]. The following antibodies were used for staining: CD19-APC, B220 (CD45R)-FITC, IgM biotin, IgD-PE, and Streptavidin-APC/Cy7 (BD Biosciences, USA). Data were collected by a flow cytometer (BriCyteE6, Mindray Bio- Medical Electronics Co. Ltd., Shenzhen, China) and analyzed using FlowJo Version 10.1 software. For the analysis of tumor-associated macrophages, cell suspensions of lymph nodes were obtained by grinding and filtering tissues through $0.4-\mu \mathrm{m}$ cell strainers (BD Biosciences, USA) in PBS. Cells were then transferred to a fresh tube for centrifugation at $1000 \times g$ for $5 \mathrm{~min}$. The cell pellet was incubated in red blood cell lysis buffer (Lonza) for $1 \mathrm{~min}$ at room temperature, diluted in PBS, and centrifuged for $1000 \times g$ for $5 \mathrm{~min}$. The cell pellet was incubated with fluorescent-conjugated antibodies (dilution 1:50 in PBS) for $15 \mathrm{~min}$ at $4{ }^{\circ} \mathrm{C}$ in the dark, washed, and analyzed by flow cytometry. The following antibodies were used: F4/80 (6F12) from Miltenyi; CD11b (M1/70.15.5) and Gr1 (RB6-8C5) from BD Pharmingen.

\subsection{Statistical Analyses}

Statistical analysis was performed by the two-tailed unpaired Student's $t$ test using the GraphPad Prism ${ }^{\circledR}$ software package. Statistical significance was determined by $p<0.05$ [48].

Supplementary Materials: Supplementary Materials can be found at http://www.mdpi.com/1422-0067/21/3/885/s1.

Author Contributions: E.V. designed and conducted the research, analysed the data, and wrote the manuscript; C.M. performed the histopathological experiments and assisted with the data analysis; E.I., S.M., D.M. assisted with the mice experiments; G.F. and F.T. contributed to data analysis and reviewed the manuscript; I.Q. supervised the research plan and data analysis, and reviewed the manuscript. All authors have read and agreed to the published version of the manuscript.

Funding: This study was supported by grant POR FES/FESR 2014-20-ATS ALCMEONE cup J18C17000610006 to I.Q.; MIUR-PRIN 2017MHJJ55_002 to I.Q.; EU project “PON-RI2014-2020” to D.M.; "PON-RI2014-2020-cup F67C18000200006 to S.M.

Conflicts of Interest: The authors declare no conflict of interest. The funders had no role in the design of the study; in the collection, analyses, or interpretation of data; in the writing of the manuscript, or in the decision to publish the results.

\section{References}

1. Balkwill, F.; Mantovani, A. Cancer and inflammation: implications for pharmacology and therapeutics. Clin. Pharmacol. Ther. 2010, 87, 401-406. [CrossRef] [PubMed]

2. Vakkila, J.; Lotze, M.T. Inflammation and necrosis promote tumour growth. Nat. Rev. Immunol. 2004, 4, 641-648. [CrossRef] [PubMed]

3. Coussens, L.M.; Werb, Z. Inflammation and cancer. Nature 2002, 420, 860-867. [CrossRef] [PubMed] 
4. Pollard, J.W. Tumour-educated macrophages promote tumour progression and metastasis. Nat. Rev. Cancer 2004, 4, 71-78. [CrossRef]

5. Hanahan, D.; Weinberg, R.A. The hallmarks of cancer. Cell 2000, 100, 57-70. [CrossRef]

6. Murdoch, C.; Muthana, M.; Coffelt, S.B.; Lewis, C.E. The role of myeloid cells in the promotion of tumour angiogenesis. Nat. Rev. Cancer 2008, 8, 618-631. [CrossRef]

7. Bingle, L.; Brown, N.J.; Lewis, C.E. The role of tumour-associated macrophages in tumour progression: implications for new anticancer therapies. J. Pathol. 2002, 196, 254-265. [CrossRef]

8. Ziyad, S.; Iruela-Arispe, M.L. Molecular mechanism of tumor angiogenesis. Genes Cancer 2011, 2, $1085-1096$. [CrossRef]

9. Falcomatà, M.; Bärthel, S.; Schneider, G.; Saur, D.; Veltkamp, C. Deciphering the universe of genetic context-dependencies using mouse models of cancer. Curr. Opin. Gen. Dev. 2019, 54, 97-104. [CrossRef]

10. James, Y.M. Mouse Models of Lymphoma and Lymphoid Leukemia. In Neoplastic Hematopathology: Experimental and Clinical Approaches; Jones, D., Ed.; Springer-Verlag New York, LLC: New York, NY, USA, 2010; Chapter 36; pp. 583-596.

11. Frese, K.K.; Tuveson, D.A. Maximizing mouse cancer models. Nat. Rev. Cancer. 2007, 7, 645-658. [CrossRef]

12. Carroll, P.A.; Freie, B.W.; Mathsyaraja, H.; Eisenman, R.N. The MYC transcription factor network: balancing metabolism, proliferation and oncogenesis. Front. Med. 2018, 12, 412-425. [CrossRef] [PubMed]

13. Adams, J.M.; Harris, A.W.; Pinkert, C.A.; Corcoran, L.M.; Alexander, W.S.; Cory, S.; Palmiter, R.D.; Brinster, R.L. The c-myc oncogene driven by immunoglobulin enhancers induces lymphoid malignancy in transgenic mice. Nature 1985, 318, 533-538. [CrossRef] [PubMed]

14. Harris, A.W.; Pinkert, C.A.; Crawford, M.; Langdon, W.Y.; Brinster, R.L.; Adams, J.M. The E mu-myc transgenic mouse. A model for high-incidence spontaneous lymphoma and leukemia of early B cells. J. Exp. Med. 1988, 167, 363-371. [CrossRef] [PubMed]

15. Egle, A.; Harris, A.W.; Bath, M.L.; O'Reilly, L.; Cory, S. VavP-Bcl2 transgenic mice develop follicular lymphoma preceded by germinal center hyperplasia. Blood 2004, 103, 2276-2283. [CrossRef]

16. Ruddell, A.; Mezquita, P.; Brandvold, K.A.; Farr, A.; Iritani, B.M. B lymphocyte-specific c-Myc expression stimulates early and functional expansion of the vasculature and lymphatics during lymphomagenesis. Am. J. Pathol. 2003, 163, 2233-2245. [CrossRef]

17. Baird, T.D.; Palam, L.R.; Fusakio, M.E.; Willy, J.A.; Davis, C.M.; McClintick, J.N.; Anthony, T.G.; Wek, R.C. Selective mRNA translation during eIF2 phosphorylation induces expression of IBTKalpha. Mol. Biol. Cell 2014, 25, 1686-1697. [CrossRef]

18. Luo, J.; Emanuele, M.J.; Li, D.; Creighton, C.J.; Schlabach, M.R.; Westbrook, T.F.; Wong, K.K.; Elledge, S.J. A genome-wide RNAi screen identifies multiple synthetic lethal interactions with the Ras oncogene. Cell 2009, 137, 835-848. [CrossRef]

19. Pisano, A.; Ceglia, S.; Palmieri, C.; Vecchio, E.; Fiume, G.; de Laurentiis, A.; Mimmi, S.; Falcone, C.; Iaccino, E.; Scialdone, A.; et al. CRL3IBTK regulates the tumor suppressor Pdcd4 through ubiquitylation coupled to proteasomal degradation. J. Biol. Chem. 2015, 290, 13958-13971. [CrossRef]

20. Fiume, G.; Scialdone, A.; Rizzo, F.; De Filippo, M.R.; Laudanna, C.; Albano, F.; Golino, G.; Vecchio, E.; Pontoriero, M.; Mimmi, S.; et al. IBTK Differently Modulates Gene Expression and RNA Splicing in HeLa and K562 Cells. Int. J. Mol. Sci. 2016, 17, 1711-1848. [CrossRef]

21. Albano, F.; Chiurazzi, F.; Mimmi, S.; Vecchio, E.; Pastore, A.; Cimmino, C.; Frieri, C.; Iaccino, E.; Pisano, A.; Golino, G.; et al. The expression of inhibitor of bruton's tyrosine kinase gene is progressively up regulated in the clinical course of chronic lymphocytic leukaemia conferring resistance to apoptosis. Cell Death Dis. 2018, 1, 1-10. [CrossRef]

22. Vecchio, E.; Golino, G.; Pisano, A.; Albano, F.; Falcone, C.; Ceglia, S.; Iaccino, E.; Mimmi, S.; Fiume, G.; Giurato, G.; et al. IBTK contributes to B-cell lymphomagenesis in E $\mu$-myc transgenic mice conferring resistance to apoptosis. Cell Death Dis. 2019, 10, 320. [CrossRef] [PubMed]

23. Michalak, E.M.; Jansen, E.S.; Happo, L.; Cragg, M.S.; Tai, L.; Smyth, G.K.; Strasser, A.; Adams, J.M.; Scott., C.L. Puma and to a lesser extent Noxa are suppressors of Myc-induced lymphomagenesis. Cell Death Differ. 2009, 16, 684-696. [CrossRef]

24. Frenzel, A.; Loven, J.; Henriksson, M.A. Targeting MYC-regulated miRNAs to combat cancer. Genes Cancer 2010, 1, 660-667. [CrossRef] [PubMed] 
25. Wong, H.L.; Jin, G.; Cao, R.; Zhang, S.; Cao, Y.; Zhou, Z. MT1-MMP sheds LYVE-1 on lymphatic endothelial cells and suppresses VEGF-C production to inhibit lymphangiogenesis. Nat. Commun. 2016, 7, 10824. [CrossRef] [PubMed]

26. Majumder, M.; Xin, X.; Lala, P.K. A practical and sensitive method of quantitating lymphangiogenesis in vivo. Lab Invest. 2013, 93, 779-791. [CrossRef]

27. Kakinuma, T.; Hwang, S.T. Chemokines, chemokine receptors, and cancer metastasis. J. Leukoc. Biol. 2006, 79, 639-651. [CrossRef]

28. Mira, E.; Lacalle, R.A.; Buesa, J.M.; de Buitrago, G.G.; Jimenez-Baranda, S.; Gómez-Moutón, C.; Martínez-A, C.; Mañes, S. Secreted MMP9 promotes angiogenesis more efficiently than constitutive active MMP9 bound to the tumor cell surface. J. Cell Sci. 2004, 1, 1847-1857. [CrossRef]

29. Rundhaug, J.E. Matrix metalloproteinases and angiogenesis. J. Cell Mol. Med. 2005, 9, 267-285. [CrossRef]

30. Zhang, J.; Patel, L.; Pienta, K.J. Targeting chemokine (C-C motif) ligand 2 (CCL2) as an example of translation of cancer molecular biology to the clinic. Prog. Mol. Biol. Transl. Sci. 2010, 95, 31-53.

31. Erreni, M.; Mantovani, A.; Allavena, P. Tumor-associated Macrophages (TAM) and Inflammation in Colorectal Cancer. Cancer Microenviron. 2011, 4, 141-154. [CrossRef]

32. Gabrilovich, D.I.; Ostrand-Rosenberg, S.; Bronte, V. Coordinated regulation of myeloid cells by tumours. Nat. Rev. Immunol. 2012, 22, 253-268. [CrossRef] [PubMed]

33. Kato, T.; Kato, T.; Kimura, T.; Miyakawa, R.; Tanaka, S.; Fujii, A.; Yamamoto, K.; Kameoka, S.; Hamano, K.; Kawakami, M.; et al. Clinicopathologic study of angiogenesis in Japanese patients with breast cancer. World J. Surg. 1997, 21, 49-56. [CrossRef] [PubMed]

34. Craig, M.J.; Loberg, R.D. CCL2 (Monocyte Chemoattractant Protein-1) in cancer bone metastases. Cancer Metastasis Rev. 2006, 25, 611-619. [CrossRef] [PubMed]

35. Lee, A.H.; Happerfield, L.C.; Bobrow, L.G.; Millis, R.R. Angiogenesis and inflammation in ductal carcinoma in situ of the breast. J. Pathol. 1997, 181, 200-206. [CrossRef]

36. Allavena, P.; Germano, G.; Marchesi, F.; Mantovani, A. Chemokines in cancer related inflammation. Exp. Cell Res. 2011, 10, 664-673. [CrossRef]

37. Aref, S.; Mabed, M.; Zalata, K.; Sakrana, M.; El Askalany, H. The interplay between c-Myc oncogene expression and circulating vascular endothelial growth factor (sVEGF), its antagonist receptor, soluble Flt-1 in diffuse large B cell lymphoma (DLBCL): relationship to patient outcome. Leuk. Lymphoma 2004, 45, 499-506. [CrossRef]

38. Riabov, V.; Gudima, A.; Wang, N.; Mickley, A.; Orekhov, A.; Kzhyshkowska, J. Role of tumor associated macrophages in tumor angiogenesis and lymphangiogenesis. Front. Physiol. 2014, 5, 5-75. [CrossRef]

39. Ebos, J.M.; Lee, C.R.; Cruz-Munoz, W.; Bjarnason, G.A.; Christensen, J.G.; Kerbel, R.S. Accelerated metastasis after short-term treatment with a potent inhibitor of tumor angiogenesis. Cancer Cell 2009, 15, 232-239. [CrossRef]

40. Pàez-Ribes, M.; Allen, E.; Hudock, J.; Takeda, T.; Okuyama, H.; Viñals, F.; Inoue, M.; Bergers, G.; Hanahan, D.; Casanovas, O. Antiangiogenic therapy elicits malignant progression of tumors to increased local invasion and distant metastasis. Cancer Cell 2009, 15, 220-231. [CrossRef]

41. Iaccino, E.; Mimmi, S.; Dattilo, V.; Marino, F.; Candeloro, P.; Di Loria, A.; Marimpietri, D.; Pisano, A.; Albano, F.; Vecchio, E.; et al. Monitoring multiple myeloma by idiotype-specific peptide binders of tumor-derived exosomes. Mol. Cancer. 2017, 16, 159. [CrossRef]

42. Lin, C.J.; Nasr, Z.; Premsrirut, P.K.; Porco, J.A., Jr.; Hippo, Y.; Lowe, S.W.; Pelletier, J. Targeting synthetic lethal interactions between Myc and the eIF4F complex impedes tumorigenesis. Cell. Rep. 2012, 1, 325-333. [CrossRef] [PubMed]

43. Li, X.; Li, P.; Liu, C.; Ren, Y.; Tang, X.; Wang, K.; He, J. Sinomenine hydrochloride inhibits breast cancer metastasis by attenuating inflammation-related epithelial-mesenchymal transition and cancer stemness. Oncotarget 2017, 8, 13560-13574. [CrossRef] [PubMed]

44. Li, X.; Zhang, Y.; Zheng, L.; Liu, M.; Chen, C.D.; Jiang, H. UTX is an escape from X-inactivation tumor-suppressor in B cell lymphoma. Nat. Comm. 2018, 9, 2720. [CrossRef] [PubMed]

45. Pontoriero, M.; Fiume, G.; Vecchio, E.; de Laurentiis, A.; Albano, F.; Iaccino, E.; Mimmi, S.; Pisano, A.; Agosti, V.; Giovannone, E.; et al. Activation of NF-кB in B cell receptor signaling through Bruton's tyrosine kinase-dependent phosphorylation of IkB- $\alpha$. J. Mol. Med. 2019, 97, 675-690. [CrossRef] [PubMed] 
46. Weidner, N.; Semple, J.P.; Welch, W.R.; Folkman, J. Tumor angiogenesis and metastasis—correlation in invasive breast carcinoma. N. Engl. J. Med. 1991, 324, 1-8. [CrossRef]

47. Fiume, G.; Scialdone, A.; Albano, F.; Rossi, A.; Tuccillo, F.M.; Rea, D.; Palmieri, C.; Caiazzo, E.; Cicala, C.; Bellevicine, C.; et al. Impairment of T cell development and acute inflammatory response in HIV-1 Tat transgenic mice. Sci. Rep. 2015, 5, 13864. [CrossRef]

48. Schiavone, M.; Fiume, G.; Caivano, A.; de Laurentiis, A.; Falcone, C.; Masci, F.F.; Iaccino, E.; Mimmi, S.; Palmieri, C.; Pisano, A.; et al. Design and characterization of a peptide mimotope of the HIV-1 gp120 bridging sheet. Int. J. Mol. Sci. 2012, 13, 5674-5699. [CrossRef]

(C) 2020 by the authors. Licensee MDPI, Basel, Switzerland. This article is an open access article distributed under the terms and conditions of the Creative Commons Attribution (CC BY) license (http://creativecommons.org/licenses/by/4.0/). 